\title{
Deux nouvelles espèces de Mesonyssus parasites nasicoles des Oiseaux de la famille Ardeidae
}

\author{
par Z. FEIDER et I. MIRONESCU
}

Laboratoire de Zoologie, Université «Al. I. Cuza» et Musée d'Histoire naturelle, Iasi, Roumanie

\section{Résumé.}

Les auteurs décrivent deux nouvelles espèces de Rhinonyssidae: Mesonyssus gerschi, parasite d'Ardea purpurea et Mesonyssus minuti, parasite d'Ixobrychus minutus.

\section{Summary.}

Two new species of Mesonyssus from the nasal cavity of Birds (Family Ardeidae).

The authors described two new species from Rhinonyssidae: Mesonyssus gerschi parasiting Ardea purpurea and Mesonyssus minuti parasiting Ixobrychus minutus.

Les acariens connus du genre Mesonyssus Fain, 1960, parasites nasicoles des oiseaux de la famille Ardeidae appartiennent aux trois espèces : 1) Mesonyssus belopolskii (Bregetova), 1950 (=Neonyssus belopolskii Bregetova, $1950 ;=N$. ardeae Zumpt et Till, $1955 ;=N$. marcandrei Gretillat, Capron et Brygoo, 1959) ; 2) Mesonyssus bubulci (Zumpt et Till), 1955 (=Neonyssus bubulci Zumpt et Till, 1955) ; et 3) Mesonyssus ixobrychi (Fain), 1956 (=Neonyssus ixobrychi Fain, 1956).

Parmi les huit espèces d'Ardeidae de Roumanie que nous avons examinées pour collecter des parasites nasicoles, nous avons trouvé deux espèces de Mesonyssus nouvelles pour la science. Pour une des espèces, nous proposons le nom Mesonyssus 
gerschi $\mathrm{n}$. sp. (dédié au $\mathrm{P}^{\mathrm{r}}$ Manfred Gersch de Jena), et pour l'autre espèce nous proposons le nom Mesonyssus minuti, d'après le nom de l'oiseau hôte.

Pour l'étude des espèces nouvelles, nous avons eu à notre disposition plusieurs espèces du genre Mesonyssus parasites sur les Ardeides (1).

\section{Mesonyssus gerschi n. sp.}

Parmi les six spécimens d'Ardea purpurea examinés, chez deux d'entre eux nous avons trouvé 25 spécimens d'acariens parasites, à savoir 19 femelles, 2 mâles et 4 protonymphes.

Les femelles et les nymphes étaient fixées sur le conca de la seconde chambre de la cavité nasale de l'hôte, tandis que les mâles restaient dans l'atrium, la première chambre de la cavité nasale.

Pendant l'été et l'automne, quand ont été faites les collections, la cavité nasale contenait aussi bien des parasites adultes que des nymphes.

\section{Caractères dimensionaux.}

Les dimensions de Mesonyssus gerschi sont comparées avec les dimensions de Mesonyssus belopolskii (Bregetova), 1950, qui parasite plusieurs espèces d'Ardeides (Ardea melanocephala, Ardea cinerea, Melanophoyx ardesiaca, Notophayx novaehollandiae, Notophayx picota) répandues dans divers continents.

Les dimensions comparatives de ces deux espèces sont notées dans le tableau I.

Du tableau I résulte que chez notre espèce l'idiosoma et les parties du corps sont plus courts que chez $M$. belopolskii, tandis que le bouclier génital de la femelle, la péritrème et les pattes de la seconde et de la quatrième paire sont plus longs.

Les dimensions de quelques articles des pattes sont notées dans le tableau II.

Du tableau II résulte que les coxes et les tarses sont plus longs chez la femelle et plus larges chez le mâle. Le rapport entre la longueur et la largeur des coxes et des tarses a presque toujours des valeurs plus grandes chez la femelle.

La longueur des coxes et des tarses a des valeurs plus grandes chez Mesonyssus belopolskii, et spécialement chez la femelle.

\section{Caractères morphologiques.}

Femelle. L'idiosoma est ovalaire, rétréci en arrière, avec les dimensions maxima au niveau des péritrèmes, tout comme chez $M$. belopolskii.

Le bouclier podosomal, avec le bord antérieur émoussé, les bords latéraux irrégulièrement rétrécis vers la partie postérieure et le bord postérieur excavé, présente une surface ponctuée et recouverte d'un réseau avec les mailles évidemment irrégu-

(1) Nous remercions A. Fain et R. Domrow qui nous ont mis à la disposition les espèces de comparaison. 

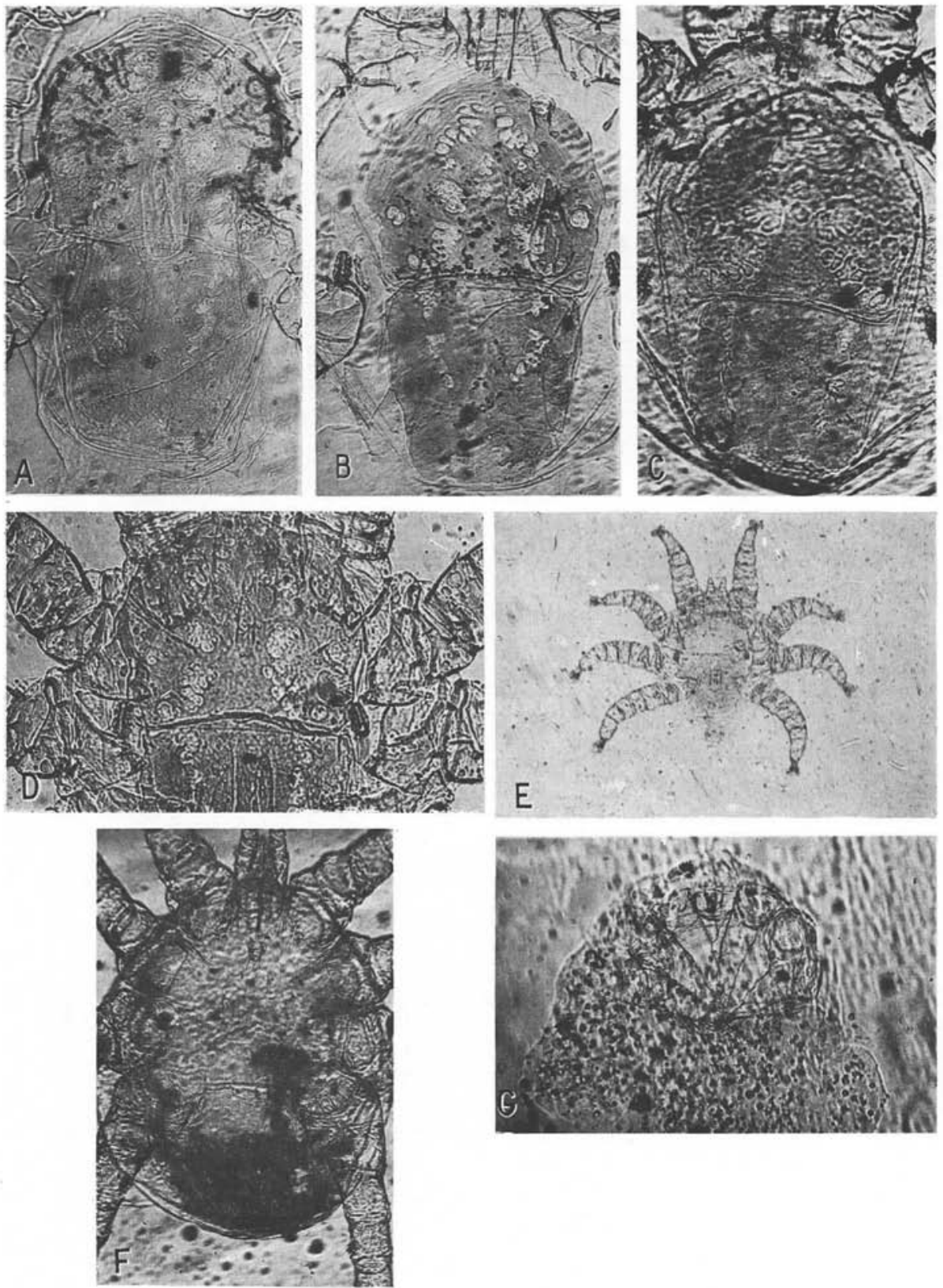

Fig. 1. - Mesonyssus gerschi n. sp. A) femelle, vue dorsale, B) mâle, vue dorsale, Mesonyssus minuti n. sp., C) femelle, vue dorsale, D) mâle, E) deutonymphe, F) deutonymphe mâle, G) larve. 


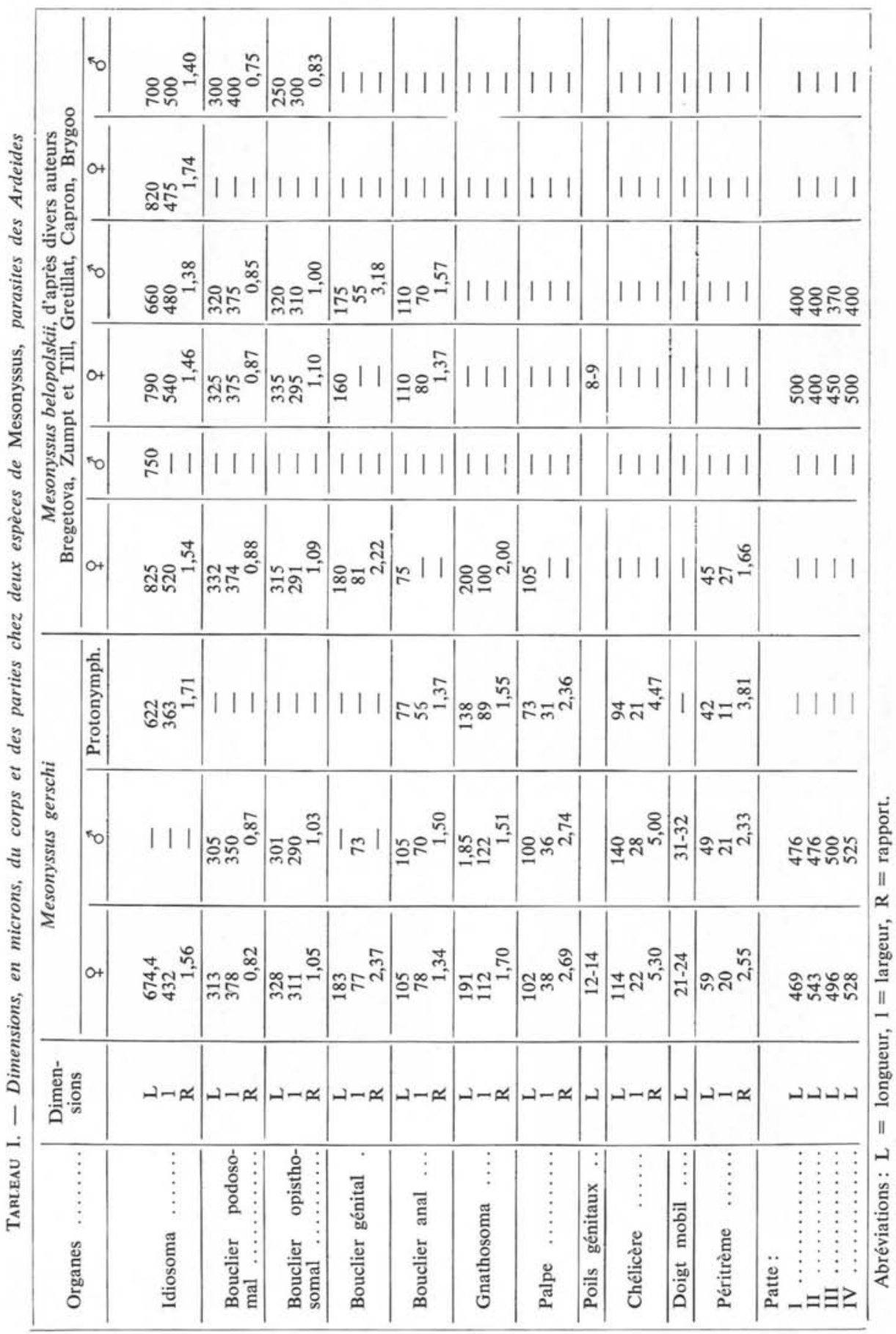




\begin{tabular}{|c|c|c|c|c|c|c|}
\hline \multirow{2}{*}{ 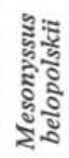 } & o+ & $\cong 1$ & $\underset{g}{ \pm} \mid 1$ & $\cong 11$ & $\underline{6} \mid 1$ & 1111 \\
\hline & ${ }^{\circ}$ & $\cong 11$ & సे 11 & సి | 1 & \& 11 & 1111 \\
\hline \multirow{3}{*}{ 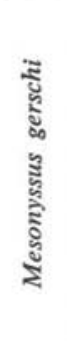 } & 율 & 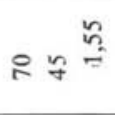 & $\tilde{\infty}=\stackrel{\tilde{b}}{-}$ & $\infty \pi \stackrel{\infty}{\infty}$ & $N \overline{\bar{J}}$ & ๓ำ ซ \\
\hline & O+ & ปே & m $\approx$ 突 & 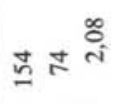 & ్ㅠㄱ & $\curvearrowleft \infty \varnothing \infty$ \\
\hline & 8 & $\tilde{g} \stackrel{g}{q}$ & 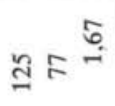 & $\stackrel{2}{y}=\stackrel{\hat{b}}{=}$ & g: & 1111 \\
\hline \multicolumn{2}{|c|}{ 它造 } & $\Perp ー \simeq$ & \lrcorner$-\simeq$ & $\leadsto ー \simeq$ & $-x$ & - \\
\hline \multicolumn{2}{|c|}{ ڤ્气 } & $\vdots$ & $=$ & $\equiv$ & $\geq$ & $\begin{array}{c}\vdots \vdots: \\
\vdots \\
-\Xi \Xi \geq\end{array}$ \\
\hline \multicolumn{2}{|c|}{$\frac{3}{\frac{3}{2}}$} & \multicolumn{4}{|c|}{$\begin{array}{l}\ddot{n} \\
\stackrel{6}{6} \\
\text { 点 }\end{array}$} & 总送 \\
\hline \multirow{2}{*}{ 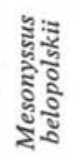 } & or & $-\infty \mid 1$ & $=11$ & $\infty \quad \mid$ & 211 & \\
\hline & "o & $=11$ & $\therefore 11$ & $\infty \quad \mid$ & $\infty 1 \mid$ & \\
\hline \multirow{3}{*}{ 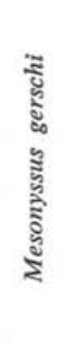 } & 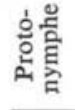 & nิ & $\Re \subseteq \stackrel{f}{\dot{q}}$ & Fミ & $g \kappa \tilde{n}$ & ॥ \\
\hline & $0+$ & $\bar{\infty} \tilde{n}^{\overline{5}}$ & $\approx \mathbb{\tilde { E }}$ & $\infty \Xi^{\stackrel{\infty}{+}}$ & aे $\stackrel{8}{0}$ & 运 \\
\hline & ro & $=\sigma^{\circ}$ & $\therefore \approx$ 웅 & $\infty \cong$ & $\infty \mathscr{\infty}_{0}^{\bar{n}}$ & $\frac{11}{5}$ \\
\hline \multicolumn{2}{|c|}{ 总呫 } & $\mu-\approx$ & $\mu-\approx$ & $\mu-\approx$ & \lrcorner$-\propto$ & 흐 \\
\hline \multicolumn{2}{|c|}{ 总 } & $\vdots$ & : & $\Xi$ & $\geq$ & 푸 \\
\hline \multicolumn{2}{|c|}{$\frac{3}{3}$} & \multicolumn{4}{|c|}{ 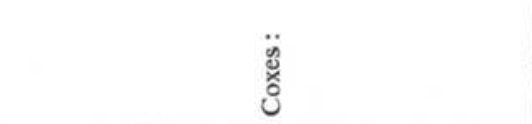 } & \\
\hline
\end{tabular}


lières (fig. 1). Dans ce réseau, quelques mailles sont transparentes et dans leur ensemble affectent une forme de lyre (fig. 2). Les mailles transparentes sont en nombre moindre et plus confondues entre elles que chez l'espèce de comparaison (fig. 3).

Le bouclier opisthosomal, trapézoïdal, tronqué ou arrondi en arrière, est recouvert d'un réseau ponctué qui présente des mailles hialines sur les parties latérales et quelquefois sur la partie postérieure.
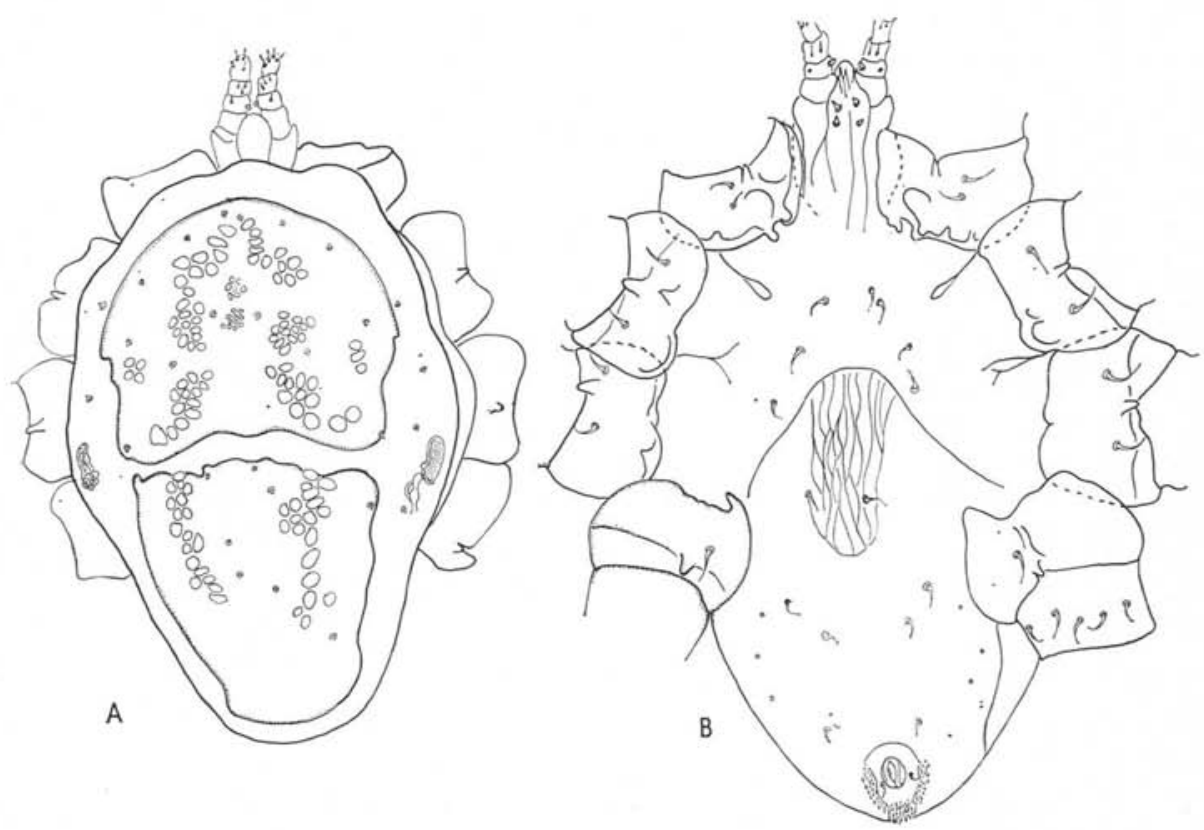

Fig. 2. - Mesonyssus gerschi, femelle. A) vue dorsale, B) vue ventrale.

Les poils dorsaux, en nombre de six paires sur le bouclier podosomal, de quatre paires sur les bords de l'idiosoma, sont petits et ponctiformes.

Sur la partie ventrale manque le bouclier sternal, mais on observe le bouclier génital et le bouclier anal. Le bouclier génital a les bords latéraux élargis vers la partie antérieure et le bord postérieur arrondi. Sur la surface du bouclier génital, on observe des stries irrégulières et, contrairement à l'espèce de comparaison, les poils génitaux sont fixés sur le bouclier. Derrière le bouclier génital se trouvent deux paires de pores et cinq paires de poils, à la différence de l'espèce de comparaison, où on observe seulement deux paires de poils.

Le bouclier anal, ovalaire, avec l'anus placé en avant de la moitié du champ claire, est pourvu d'une seule paire de poils, fixés au niveau du bord postérieur de l'anus et 
d'un cribrum terminal, avec onze rangées de denticules. Sur les bords latéraux du bouclier anal se trouvent deux prolongements du cribrum avec cinq-six rangées de denticules (fig. 3, B).

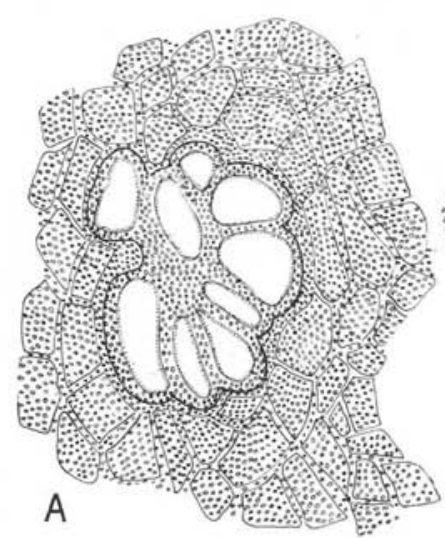

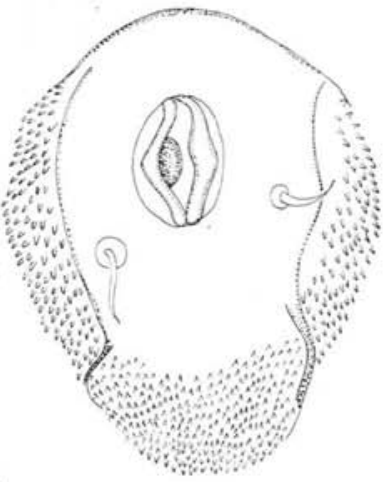

B

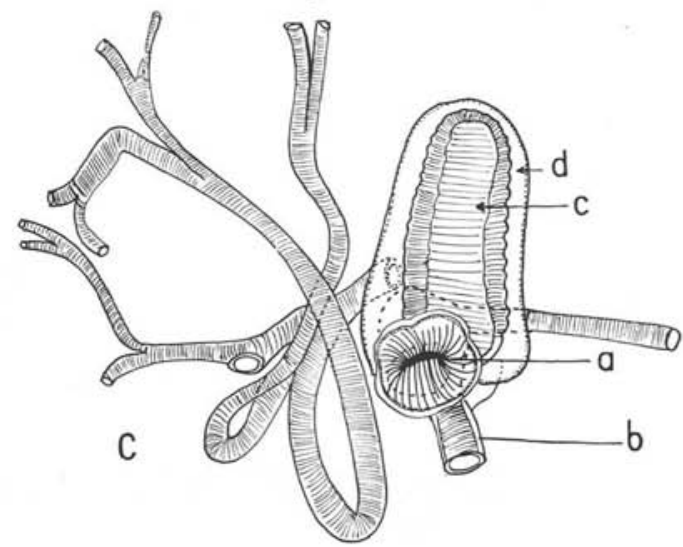

FIg. 3. - Mesonyssus gerschi, femelle. A) réseau du bouclier podosomal, B) bouclier anal, C) péritrème: a) stigme, b) trachée, c) boulier du péritrème, d) gouttière du péritrème.

Le péritrème (fig. $3, \mathrm{C}$ ) est placé sur les côtés du bouclier podosomal, au niveau de la limite entre les deux boucliers dorsaux, comme chez la sous-espèce $M$. belopolskii nycticoracis Fain, 1961, à la différence de $M$. belopolskii typica (Bregetova), 1950.

Le gnathosoma a les bords latéraux convexes et le bord postérieur droit. L'extrémité supéro-dorsale présente un tectum ovalaire dont les bords sont renforcés par des rameaux chitineux divisés dichotomiquement (fig. 4, A).

Chez les autres espèces, du genre Mesonyssus, les auteurs n'ont pas signalé l'existence du tectum. 
De même, chez une autre espèce Mesonyssus hirsutus (Feider), 1962, nous avons observé le tectum, qui est difficile à voir (fig. 4, B). On peut supposer donc que le tectum se trouve aussi chez les autres espèces du genre Mesonyssus.

Sur la partie ventrale et latérale, au voisinage de la base du palpe, on observe deux rangées formées d'une douzaine de denticules. La paire de poils gnathosomaux

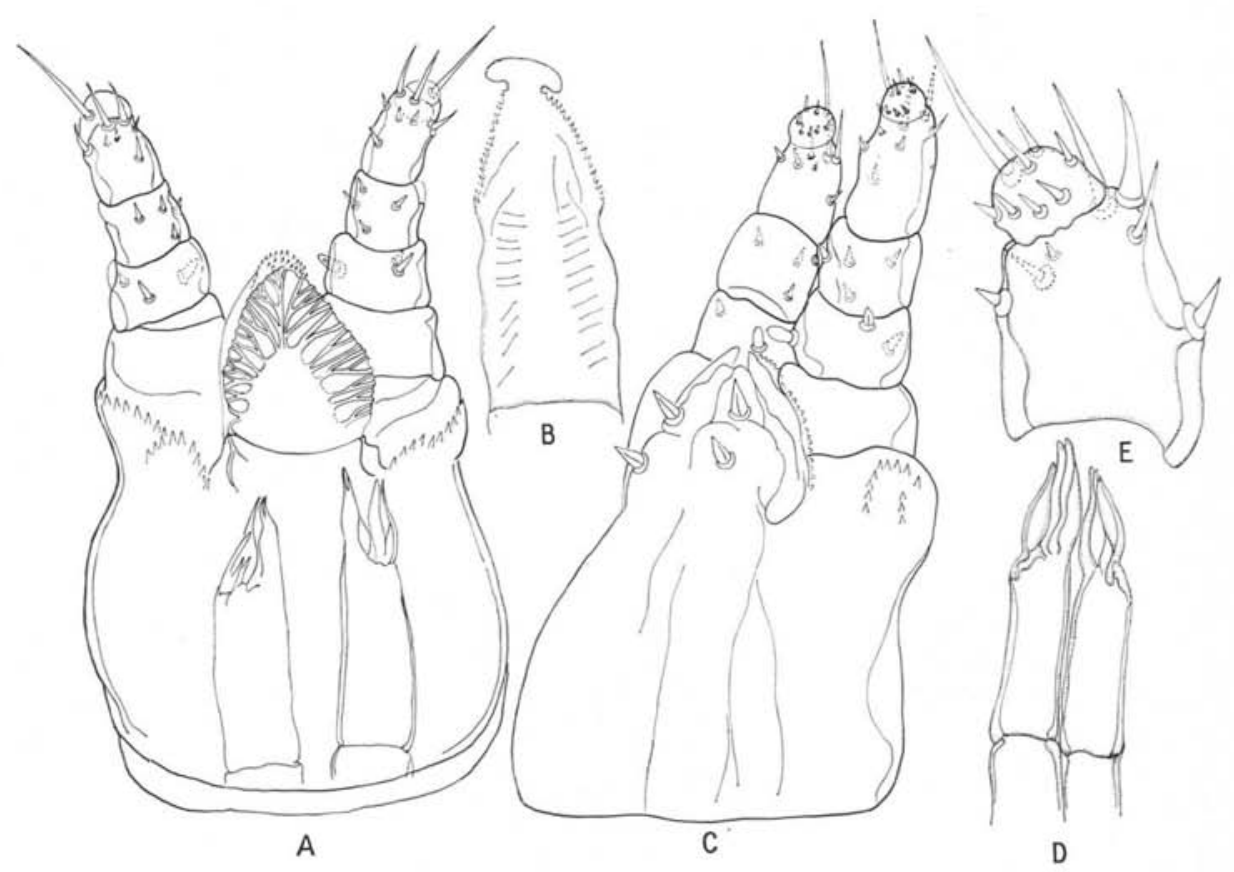

FIG. 4. - Mesonyssus gerschi, femelle. A) gnathosoma, vue dorsale, C) gnathosoma, vue ventrale, D) chélicères, E) extrémité du palpe, B) tectum de la femelle de Mesonyssus hirsutus.

et une paire de poils hypostomaux postérieurs manquent chez notre espèce. Les autres poils sont petits, forts et coniques (fig. 4, C).

Les chélicères ont le mors mobile plus court que le mors fixe, qui est divisé en deux lambeaux, dans toute sa longueur. Les mors sont membraneux et n'ont pas la possibilité de perforer le tégument (fig. 4,D). Ce caractère est une particularité du genre Mesonyssus:

Le palpe est court, robuste, avec le palpo-tarse papiliforme. Le nombre de poils du palpe est le suivant: trochanter sans poils; fémur, trois poils, dont l'interne est oviforme et les autres cunéiformes; génual, quatre poils cunéiformes, et tibia, sept poils, dont six disposés en un anneau inférieur. Dans cet anneau se trouvent deux 
poils longs supérieurs, deux poils petits latéraux et deux poils inférieurs, tandis que le septième poil est placé derrière la partie dorsale (fig. 4, E).

Le palpo-tarse, dirigé en bas, présente au-dessus, vers son extrémité, un long poil central, au-dessous duquel se trouvent respectivement trois et quatre poils plus courts et plus minces, ayant le sommet aigu.

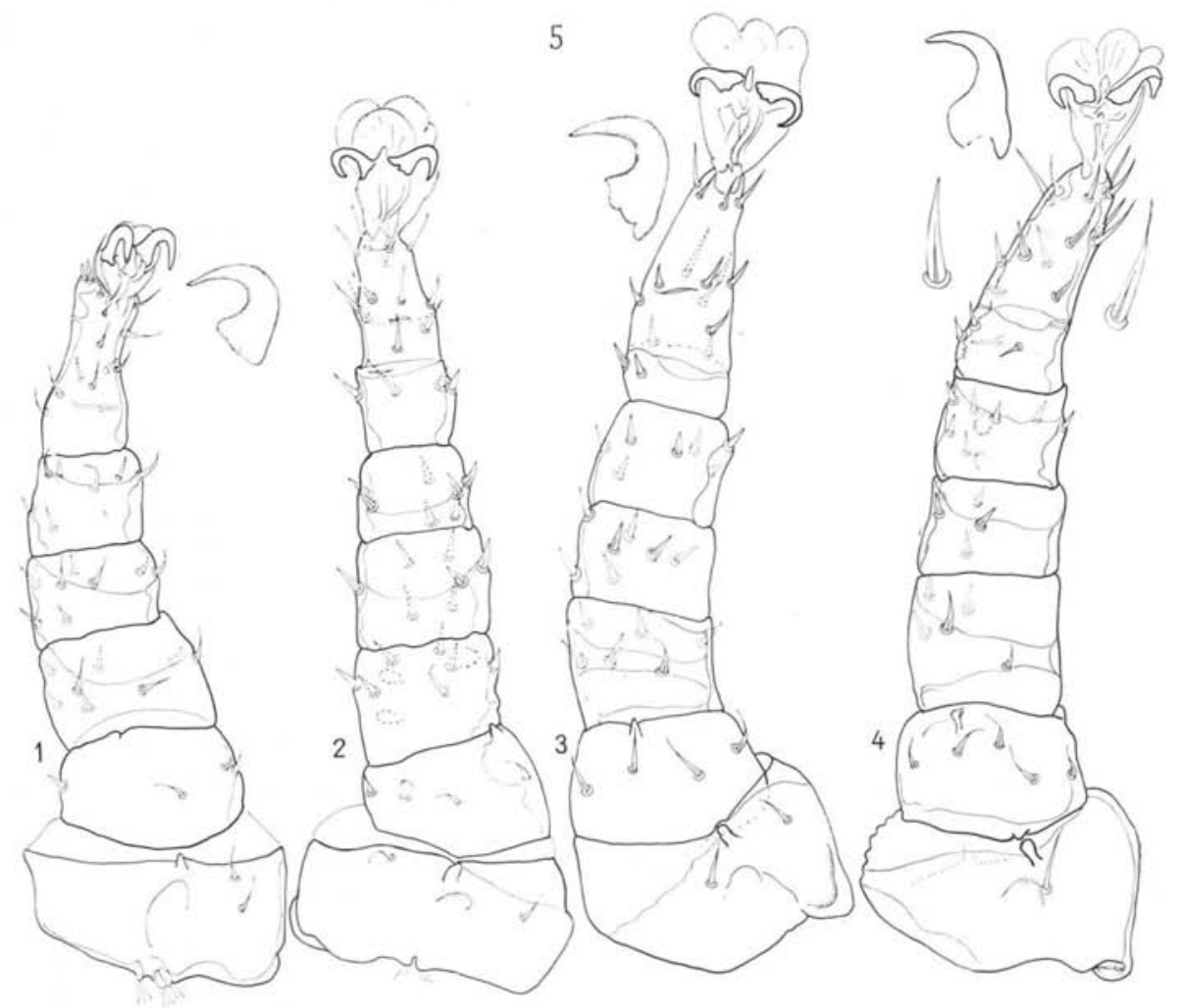

FIG. 5. - Mesonyssus gerschi, femelle. Pattes.

Les pattes ont les griffes très courbées et l'apotèle et l'empodium de la première paire très courts.

Relativement à la morphologie des coxes, on doit remarquer que, outre les deux apophyses articulaires, dont une dorsale et l'autre ventrale, on observe une petite échancrure sur le bord interne de la coxe. Chez $M$. belopolskii, cette échancrure est très profonde. Sur chaque tarse s'observe une lyrifissure basale, très large, précédée d'une autre plus courte, située sur la partie dorsale (fig. 5). 
Le nombre et la topographie des poils des pattes sont notés dans le tableau III. Dans ce tableau, on suppose que les pattes sont dirigées transversalement en dehors.

Les poils de la partie dorsale sont notés au-dessus de la ligne fractionnaire, les poils ventraux au-dessous de cette ligne, les poils du côté antérieur à gauche de la ligne et ceux du côté postérieur à droite de la ligne.

Du tableau III, on observe qu'il y a une grande variation interspécifique du nombre des poils sur les articles des pattes. Le plus grand nombre des poils se trouve sur les tarses et la plus grande amplitude de variation du nombre se trouve sur le fémur et le génual.

Chez les deux sexes, on observe des différences numériques entre les poils des pattes, à savoir sur les trois dernières paires chez $M$. gerschi, sur la première et la troisième paire chez $M$. belopolskii et sur les quatre paires de pattes chez $M$. hirsutus.

Relativement à la forme des poils, le tarse a des poils aiguisés et très allongés, tandis que les autres articles ont des poils coniques sur la partie dorsale, et des poils pourvus d'un sommet flagelliforme sur la partie ventrale (fig. 5).

Sur la première patte, les poils du bouton sensitif manifestent une spécialisation intéressante, en relation probablement avec leurs fonctions chémo-réceptoires. Le bouton sensitif est une formation qu'on observe chez toutes les espèces de Rhinonyssides, et en général chez les Gamasides. Chez l'espèce de Mesonyssus, où les griffes de la première patte ont acquis une nouvelle fonction, d'organe perforateur, la spécialisation du bouton sensitif est plus avancée. Cette spécialisation est manifestée tant par la position du bouton, disposé perpendiculairement sur l'axe du tarse, que par la structure des poils (fig. 6, A, B).

En effet, selon les observations microscopiques, le bouton sensitif est formé de plusieurs catégories de poils: 1) Un poil corunculé avec un lumen large, dans la moitié distale duquel on observe des proéminences en forme de coruncules dirigés vers le lumen. Ce poil peut être considéré comme un solénidion (fig. 6, C). 2) Un poil enfocé, avec la base fixée sous le niveau du tégument et l'extrémité émoussée, dont le lumen étroit arrive jusqu'aux deux extrémités. Autour de la base du poil, on observe une cavité hémisphérique dont le diamètre est deux fois plus large que le poil même (fig. 6, D). 3) Un poil allongé, aigu, dont le lumen devient de plus en plus large vers la base (fig. 6, E). 4) Un poil court, émoussé, avec l'extrémité arrondie et avec un lumen étroit (fig. 6, F). 5) Un poil aigu, avec l'extrémité aiguë et le lumen invisible (fig. 6, G).

Les poils du bouton sensitif ont une topographie caractéristique pour chaque espèce. Chez $M$. gerschi, la disposition est la suivante: quatre poils sont fixés dans l'aire centrale du bouton, cinq poils se trouvent dans l'aire périphérique ainsi qu'on observe dans le schéma, où les poils sont notés dans le quatre cadranes (fig. $7, \mathrm{Mg}$ ).

De même, chez une autre espèce $M$. hirsutus, le bouton sensitif est disposé dans un plan perpendiculaire sur l'axe du tarse et présente les cinq types de poils mentionnés, avec une disposition topographique similaire. Toutefois, en comparaison avec l'espèce précédente, le bouton sensitif diffère par l'absence du poil court émoussé (fig. 7, Mh). 


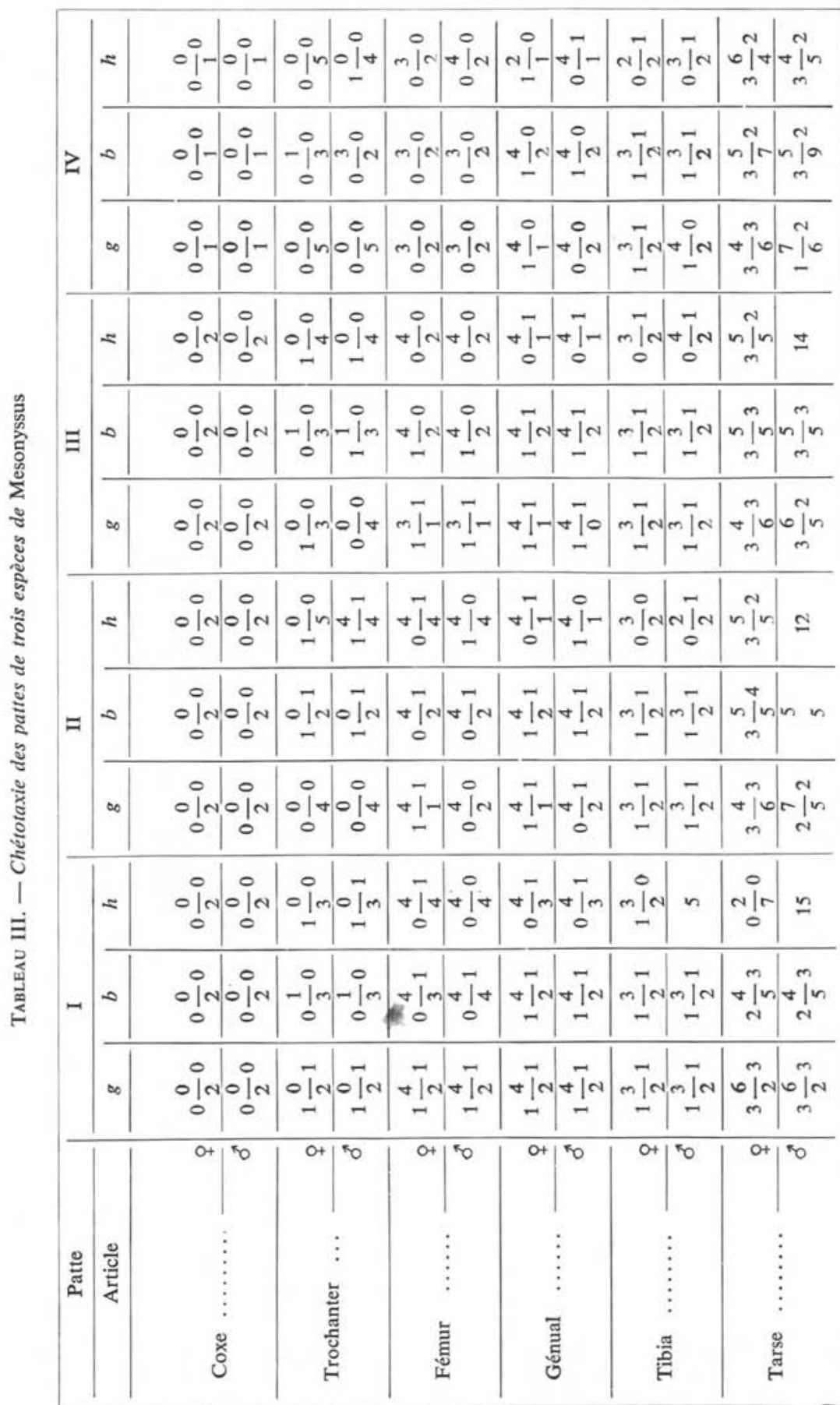




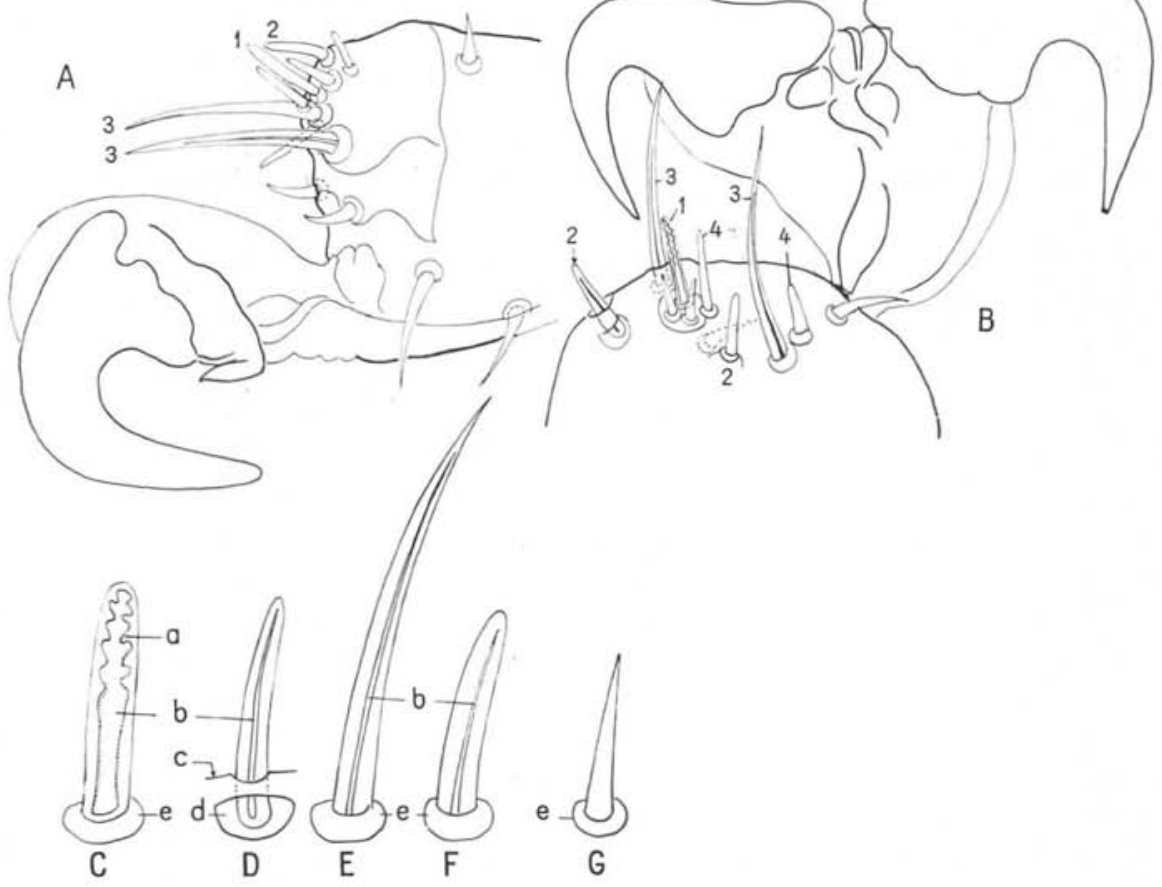

FIG 6. - Menonyssus gerschi, femelle, bouton sensitif. A) vue latérale, B) vue dorsale. Poils du bouton sensitif, C) poil corunculé, D) poil enfocé, E) poil allongé, F) poil court émoussé, G) poil court aigu : a) coruncule, b) lumen du poil, c) tégument, d) cavité de la base du poil, e) socle.

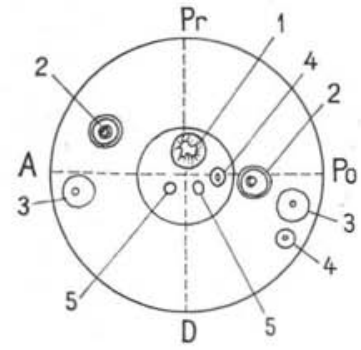

$\mathrm{Mg}$

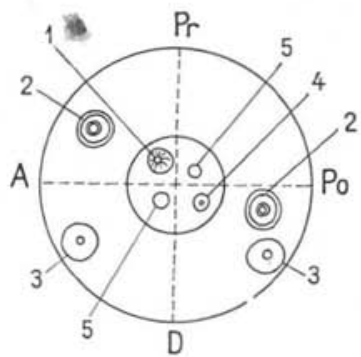

Mh

FIG. 7. - Schéma des poils du bouton sensitif chez $\mathrm{M}$. gerschi (Mg.) et $\mathrm{M}$. hirsutus (Mh). A : antérieur, D: distal, $\mathrm{Po}$ : postérieur, $\operatorname{Pr}$ : proximal. 1: poil corunculé, 2: poil enfocé, 3: poil allongé, 4: poil court émoussé, 5: poil aigu. 
Mâle. Le bouclier podosomal présente l'extrémité antérieure en forme d'angle émoussé, tandis que le bouclier opisthosomal présente l'extrémité postérieure plus arrondie. Par ces caractères, ils diffèrent de la forme des boucliers dorsaux de la femelle.

Le nombre des poils, sur le bouclier podosomal, est le même que chez la femelle, tandis que le nombre des poils sur le bouclier opisthosomal est plus grand (huit paires) (fig. $1, \mathrm{~B} ; 8, \mathrm{~A})$.
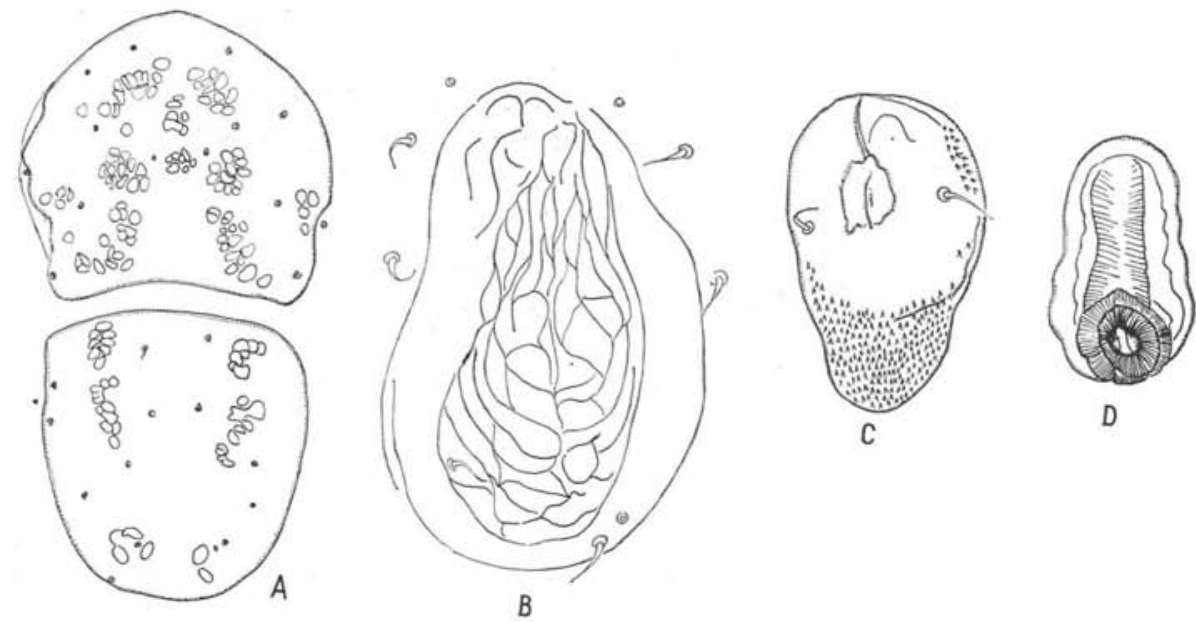

Fig. 8. - Mesonyssus gerschi, mâle. A) vue dorsale, B) bouclier ventral, C) bouclier anal, D) péritrème.

Sur la partie ventrale, on observe le bouclier ventral piriforme, réticulé à sa partie centrale, sur lequel se trouve une paire de poils effilés, fixés sur la partie postérieure (fig. 8, B).

Le bouclier anal, plus étroit, a le cribrum plus étalé vers l'orifice anal. Les poils anaux se trouvent au niveau du bord postérieur de l'orifice anal (fig. 8, C).

Le gnathosoma, de même que chez la femelle, présente les denticules antérolatérales et le même nombre de poils hypostomaux (fig. 9, A).

Les doigts des chélicères ont l'extrémité divisée en trois-quatre lobes minces (fig. 9, B).

Le palpe présente sur le fémur un poil épaissi, mais plus effilé à l'extrémité que chez la femelle. Le palpo-tarse présente neuf poils, dont un long, un court et aigu, et les autres poils papilliformes (fig. 9, C).

La première paire de pattes présente le bouton sensitif avec le même nombre et la même structure des poils que la femelle (fig. 9, D). 
Protonymphe. Les boucliers de la protonymphe, extraite du corps de la femelle sont peu visibles, à l'exception du bouclier anal, qui présente un cribrum postérieur, simple, pourvu de quatre rangées de dents et d'une seule paire de poils (fig. 10, A, B). Le cribrum triple sur la partie postérieure et sur les côtés est donc une acquisition de l'adulte.

Le gnathosoma, les mors des chélicères ressemblent à l'adulte (fig. 10, C, D). Le palpo-tarse présente seulement sept poils au lieu de huit comme l'adulte.

Sur le tarse de la première paire de pattes, le bouton sensitif présente seulement six poils (fig. 11, A). La quatrième paire de pattes, avec une seule lyrifissure sur le tarse, a un nombre plus réduit de poils sur le tarse et le tibia, tandis que le génual a le même nombre de poils que l'adulte (fig 11, B).

Larve. La larve que nous avons observée est en réalité seulement une stase embryonnaire, vu que nous l'avons extraite du corps de la femelle, qui est nymphipare. C'est pourquoi les boucliers ne sont pas visibles et que les poils du corps, avec quelques exceptions, ne sont pas présents. Toutefois, quelques poils du gnathosoma, du palpo-tarse et des extrémités des tarses I, c'est-à-dire les poils sensitifs en nombre de six et un poil présensitif, sont présents (fig. 12, A, B).

On peut remarquer que chez les stases préadultes le nombre de poils du bouton sensitif ne dépasse pas le nombre des poils du palpo-tarse, et que chez l'adulte on observe le contraire.

$\mathrm{Ce}$ fait peut nous indiquer que la spécialisation complète de la première paire de pattes, comme organe de lésion de la muqueuse, se fait tardivement dans l'ontogénie.

Holotype et paratypes, femelles, allotype et paratypes mâles, et paratypes protonymphes chez les auteurs. Terra typica: Delta du Danube. Date : 30 juin 1968. Hôte : Ardea purpurea.

\section{Mesonyssus minuti n. sp.}

Parmi les sept spécimens d'Ixobrychus minutus (2) examinés, nous avons trouvé six spécimens parasités par 19 spécimens de $M$. minuti, dont deux femelles, deux deutonymphes et deux protonymphes sur le conca, et treize mâles localisés dans l'atrium.

\section{Caractères dimensionaux.}

Les dimensions de cette espèce sont notées dans le tableau IV.

Du tableau IV résulte que les dimensions de l'idiosoma, des boucliers et des appendices sont moindres chez $M$. minuti en comparaison avec $M$. gerschi, et même assez petites que chez $M$. belopolskii.

(2) Nous remercions C. Mândru pour les spécimens d'Ixobrychus minutus mis à notre disposition. 


\section{Caractères morphologiques.}

Femelle. Le bouclier podosomal diffère du bouclier de $M$. gerschi et de $M$. ixobrichi Fain, 1956, par la forme régulière du bord et par la présence d'une paire de poils longs à côté des poils rudimentaires.

Le bouclier opisthosomal avec le bord régulier est moins allongé que chez les espèces de comparaison. En outre, il présente trois paires de poils longs à côté des poils ponctiformes.

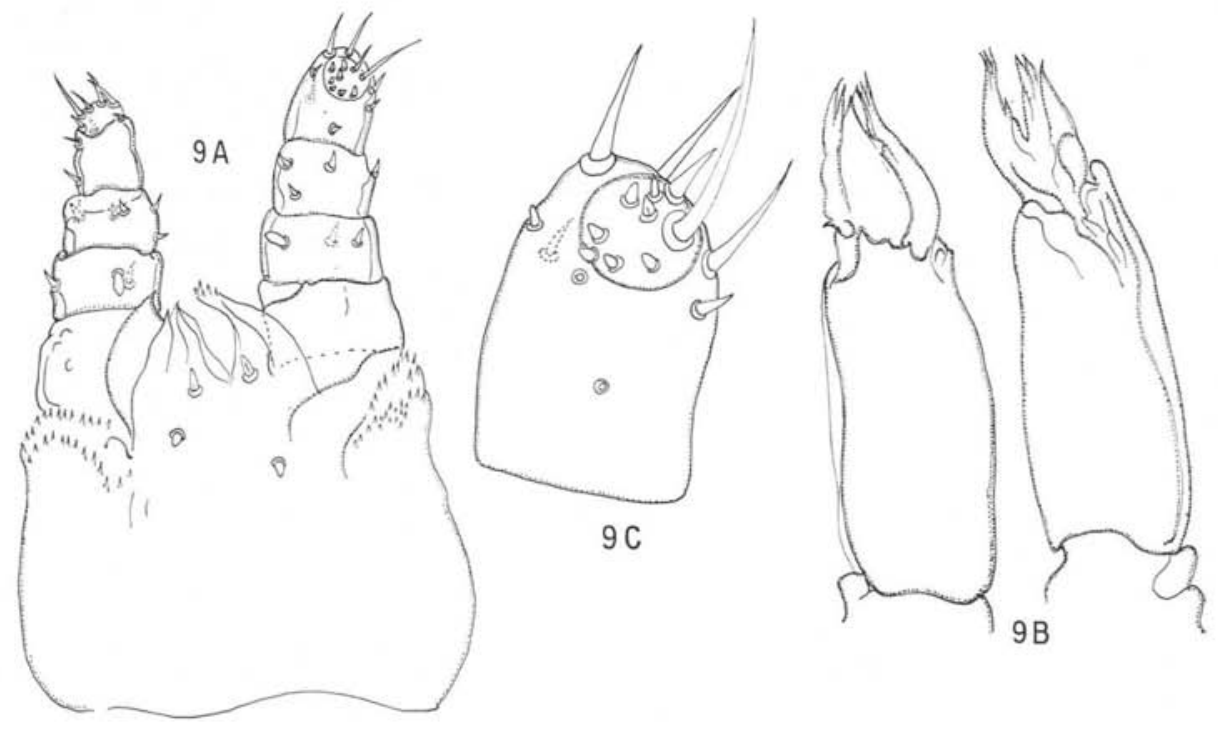

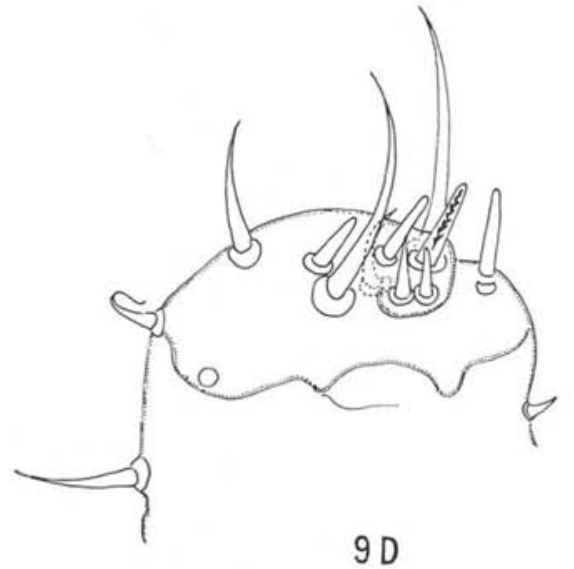

FIG. 9. - Mesonyssus gerschi, mâle. A) gnathosoma, vue ventrale, B) chélicères, C) palpotarse, D) bouton sensitif. 


\begin{tabular}{|c|c|c|c|c|c|c|c|c|c|c|c|c|}
\hline 总竞 & 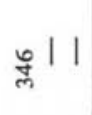 & 卢 1 | & $\stackrel{\infty}{2} \mid$ & $g^{\prime} \mid 1$ & บำ & ๓ะ๐ & พับฮ์ & б) & ஜே & มูำ & $a^{\frac{m}{i}}$ & 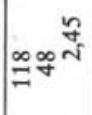 \\
\hline 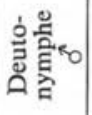 & ूू| & 앙 1 & $\frac{m}{z} \mid 1$ & $\approx$ & อีู่์ & ชะู่ & 웜 & 성 & "సतి & छूñ & छำส & సిగ్ల \\
\hline ro & สู | & ন্ডে & $\frac{6}{4} 1$ & $n_{n}^{1} 1$ & 등 & Nmon & $\infty \underbrace{n}$ & $\operatorname{Ros}$ & $\overline{\operatorname{n}} \stackrel{\stackrel{\infty}{=}}{=}$ & $\operatorname{og}^{2} \approx$ & İn & mñ \\
\hline or & 111 & 111 & 1 & $\frac{a}{n} \mid 1$ & $\operatorname{sem}^{\vec{b}}$ & 룽 & অ. & 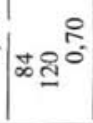 & सสูปี & $\cong \approx \frac{n}{N}$ & 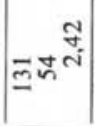 & gn \\
\hline 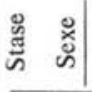 & 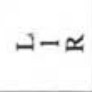 & -ーー & 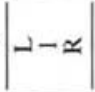 & $\triangle-\approx$. & $-\lambda-\approx$ & 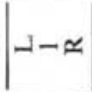 & $\Lambda-\approx$ & I & $-2-\simeq$ & エ & $\Lambda ー \simeq$ & U \\
\hline 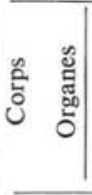 & 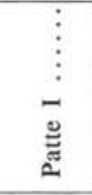 & 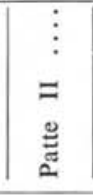 & $\begin{array}{l}\Xi \\
\cong \\
\Xi\end{array}$ & 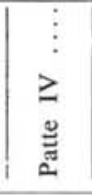 & $\begin{array}{r}\vdots \\
\vdots \\
\vdots \\
\text { o } \\
\text { ¿ }\end{array}$ & $\begin{array}{c}\vdots \\
\vdots \\
= \\
\text { ठิ }\end{array}$ & $\begin{array}{l}\vdots \\
\vdots \\
\Xi \\
0 \\
0 \\
0\end{array}$ & $\begin{array}{l}\vdots \\
\vdots \\
\geq \\
\dot{y} \\
0\end{array}$ & 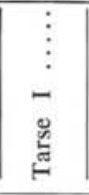 & 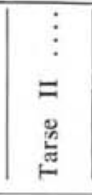 & 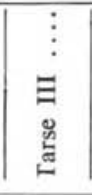 & 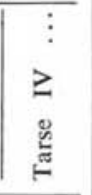 \\
\hline 宫䓂 & బูస్త్ & 111 & 111 & 111 & \&: & me & ह & nू & గ $\begin{array}{r}\infty \\
\text { रें }\end{array}$ & -11 & 111 & 1 \\
\hline 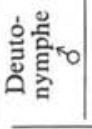 & 웅 & लైలో & 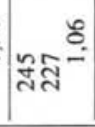 & 111 & Еดั & $\mathcal{\mathcal { V }} \mid$ & ミัษ & চ & - & 111 & 111 & 1 \\
\hline${ }^{\circ}$ & กู่ะ & $\frac{\infty}{m} \stackrel{\&}{\infty}$ & ลิธ్ & ミะ & ลละ & ทักิ & ๓ & 주 & बूल् & 111 & 111 & 1 \\
\hline ot & ํํำ & 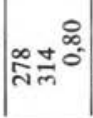 & 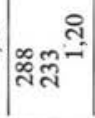 & กูกู & $\ln 1$ & $\mathcal{2} \mid 1$ & & $\infty \stackrel{0}{\infty} \underset{\sim}{ }$ & ఐ & ô 11 & 011 & $\frac{n}{\grave{I}}$ \\
\hline 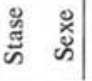 & 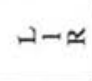 & $\Lambda-\simeq$ & ニー & 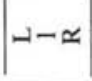 & \lrcorner$-\simeq$ & $\Lambda-\approx$ & $\Lambda-\simeq$ & $\Lambda ー \simeq$ & $\Lambda-\simeq$ & ـ & $-\sim-\simeq$ & , \\
\hline 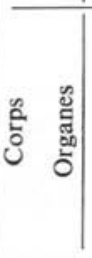 & 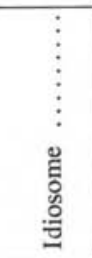 & 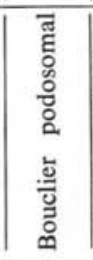 & 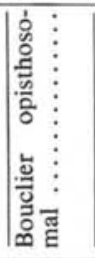 & 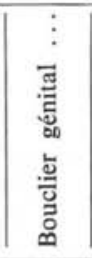 & 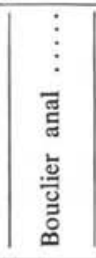 & $\begin{array}{c}\vdots \\
\vdots \\
\vdots \\
\text { 芯 } \\
\text { 总 }\end{array}$ & 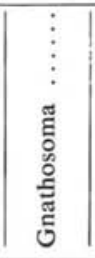 & 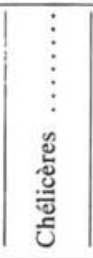 & 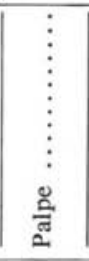 & 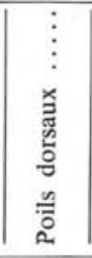 & 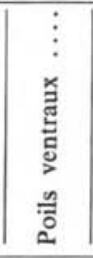 & 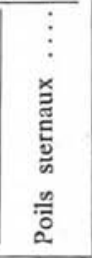 \\
\hline
\end{tabular}


A l'extérieur des boucliers dorsaux, on observe cinq paires de poils ponctiformes (fig. 13, A).

Sur la partie ventrale manque le bouclier sternal, qui est présent chez $M$. ixobrichi. Les poils sternaux sont effilés comme chez $M$. gerschi. Le bouclier génital est moins

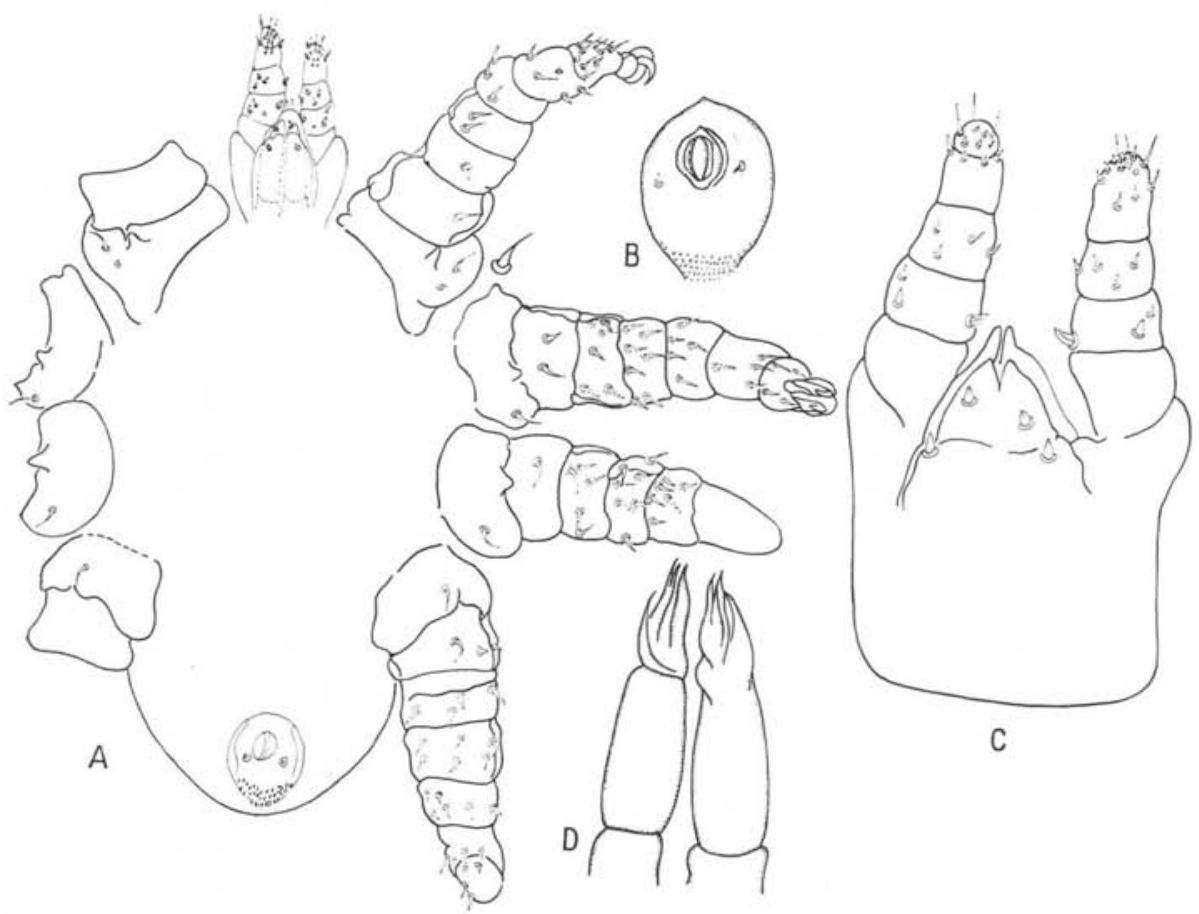

Fig. 10. - Mesonyssus gerschi. Protonymphe dans la peau de la larve. A) vue ventrale, B) bouclier anal, C) gnathosoma, D) chélicères.

allongé que chez les deux espèces de comparaison et en outre présente l'extrémité postérieure en forme d'angle émoussé.

Parmi les cinq paires de poils opisthosomaux, dans la première et les deux dernières paires, les poils sont allongés et effilés, tandis que chez $M$. gerschi les premières paires et la dernière paire ont des poils effilés.

Le bouclier anal présente les poils fixés au niveau du bord antérieur de l'orifice anal (fig. 13, B).

Les péritrèmes sont plus étroits que chez $M$. gerschi (fig. 13, C).

Le gnathosoma est étroit et dépourvu des denticules de l'extrémité antéro-latérale qu'on observe chez $M$. gerschi. Les poils hypostomaux manquent, mais on observe les 
trois paires de poils gnathostomaux, dont la première paire est forte et conique. Par les poils gnathostomaux, $M$. minuti diffère de $M$. gerschi et de $M$. ixobrichi.

Sur le second article du palpe, on observe une paire de poils coniques forts qui manquent chez $M$. gerschi, tandis que chez $M$. ixobrichi on observe seulement les poils internes qui ont une forme piriforme ovalaire (fig. 14, A).

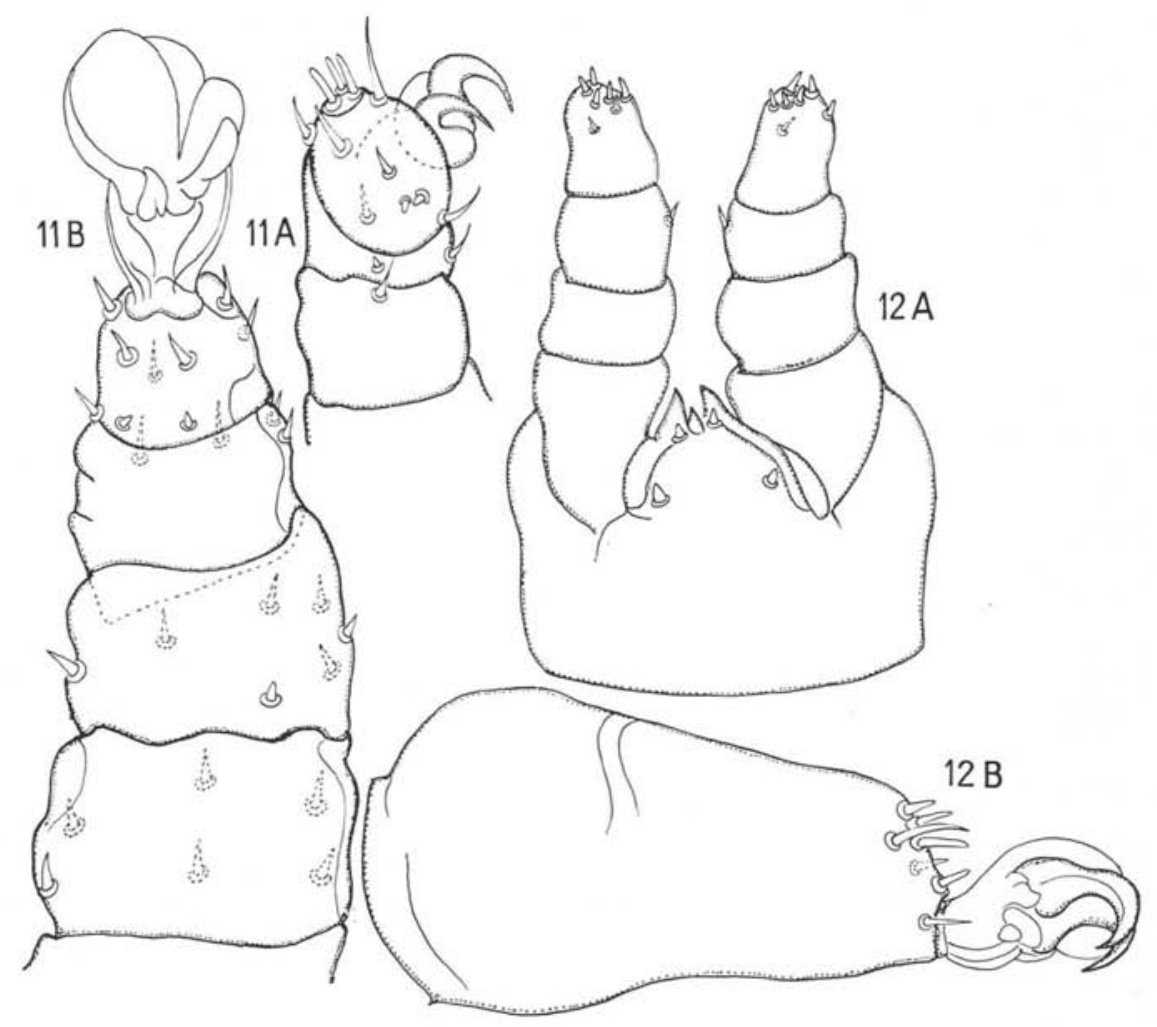

Fig. 11. - Mesonyssus gerschi. Protonymphe. A) tarse 1, B) patte 4.

FIG. 12. - Mesonyssus gerschi. Larve. A) gnathosoma, B) tarse 1.

Les chélicères ont le mors fixe, dépourvu de fente longitudinale (fig. 14, B).

Le palpo-tarse est pourvu de huit poils, dont cinq ponctiformes, un poil allongé et deux poils longs, tandis que chez $M$. gerschi aucun poil n'est ponctiforme et un seul poil est allongé.

Le nombre et la disposition des poils sur les pattes, représentés d'après la même manière que chez l'espèce précédente, sont notés dans le tableau V. 
TABleau V. - Chétotaxie des pattes chez Mesonyssus minuti

\begin{tabular}{|c|c|c|c|c|c|c|c|c|}
\hline & Patte & Sexe & Coxe & Trochanter & Fémur & Génual & Tibia & Tarse \\
\hline \multirow{2}{*}{\multicolumn{2}{|c|}{ I }} & $q$ & $0 \frac{0}{2} 0$ & $0 \frac{0}{4} 0$ & $1 \frac{4}{2} 1$ & $1 \frac{4}{2} 1$ & $1 \frac{3}{2} 1$ & $3 \frac{4}{3} 1$ \\
\hline & & 8 & $0 \frac{0}{2} 0$ & $0 \frac{1}{3} 0$ & $1 \frac{4}{1} 1$ & $1 \frac{4}{2} 1$ & $1 \frac{3}{2} 1$ & \\
\hline \multirow{2}{*}{\multicolumn{2}{|c|}{ II }} & 우 & $0 \frac{0}{2} 0$ & $0 \frac{0}{4} 0$ & $1 \frac{4}{1} 1$ & $1 \frac{4}{2} 1$ & $1 \frac{4}{1} 1$ & $3 \frac{4}{8} 2$ \\
\hline & & 8 & $0 \frac{0}{2} 0$ & $0 \frac{0}{4} 0$ & $1 \frac{4}{1} 1$ & $1 \frac{4}{2} 1$ & $1 \frac{3}{2} 1$ & \\
\hline \multirow{2}{*}{\multicolumn{2}{|c|}{ III }} & q & $0 \frac{0}{2} 0$ & $0 \frac{0}{4} 0$ & $1 \frac{4}{1} 1$ & $1 \frac{4}{2} 1$ & $1 \frac{3}{2} 1$ & $1 \frac{7}{6} 3$ \\
\hline & & 6 & $0 \frac{0}{2} 0$ & $0 \frac{0}{4} 0$ & $1 \frac{4}{1} 1$ & $1 \frac{4}{2} 1$ & $1 \frac{3}{2} 1$ & $3 \frac{5}{5} 3$ \\
\hline \multirow{2}{*}{\multicolumn{2}{|c|}{ IV }} & $q$ & $0 \frac{0}{1} 0$ & $0 \frac{0}{5} 0$ & $1 \frac{3}{1} 1$ & $1 \frac{4}{1} 1$ & $1 \frac{3}{2} 1$ & $2 \frac{5}{6} 3$ \\
\hline & & $\sigma^{\circ}$ & $0 \frac{0}{1} 0$ & $0 \frac{0}{5} 0$ & $0 \frac{3}{2} 0$ & $1 \frac{4}{2} 1$ & $1 \frac{3}{2} 1$ & $2 \frac{7}{5} 2$ \\
\hline
\end{tabular}

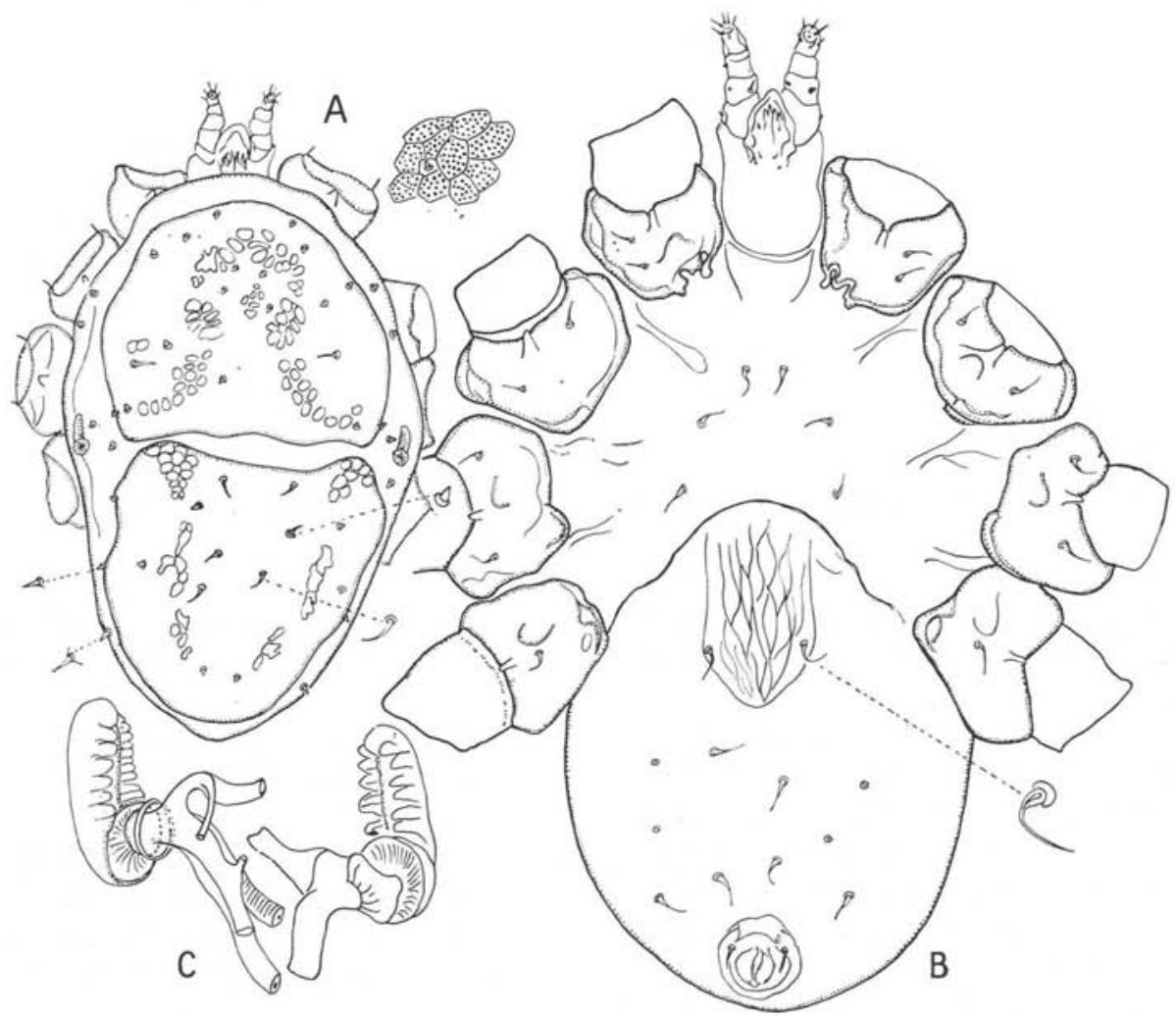

FIG. 13. - Mesonyssus minuti, femelle. A) vue dorsale, B) vue ventrale, C) péritrème et tronc trachéen. 
Du tableau résulte que Mesonyssus minuti diffère de $M$. gerschi par le nombre des poils sur les tarses I, II et III, sur le génual II, III et IV et sur le fémur III, et par la disposition des poils sur le tarse IV. Les deux sexes diffèrent par le nombre des poils sur le tarse III, sur le génual IV, sur le fémur I et IV et sur le trochanter I, et par la disposition des poils sur le tarse IV et sur le tibia II.

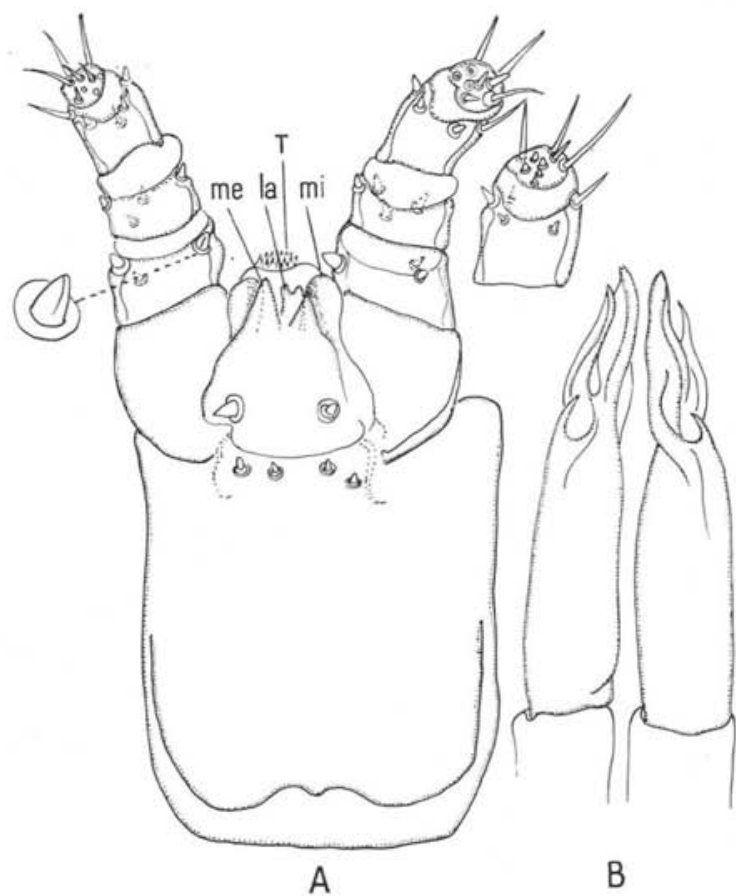

FIG. 14. - Mesonyssus minuti, femelle. A) gnathosoma, vue ventrale, B) chélicères.

On doit remarquer que $M$. minuti diffère aussi de $M$. gerschi par la subdivision du fémur IV, dont le télofémur porte tous les poils, tandis que le basifémur en est dépourvu (fig. 15).

Mâle. Le bouclier podosomal, plutôt pentagonal, présente l'angle antérieur émoussé et l'angle antéro-latéral déplacé plus en arrière que chez le mâle de $M$. gerschi. Les sept paires de poils sont papilliformes.

Le bouclier opisthosomal, avec le contour irrégulier, est rétréci vers l'extrémité postérieure. Parmi les neuf paires de poils, deux paires sont plus longues (fig. 16, A).

Le bouclier ventral est rhomboïdal et rétréci vers les deux extrémités. Les mailles du réticule sont allongées dans la direction antéro-postérieure. Les poils du bouclier sont plus courts que les poils sternaux (fig. 16, B). 


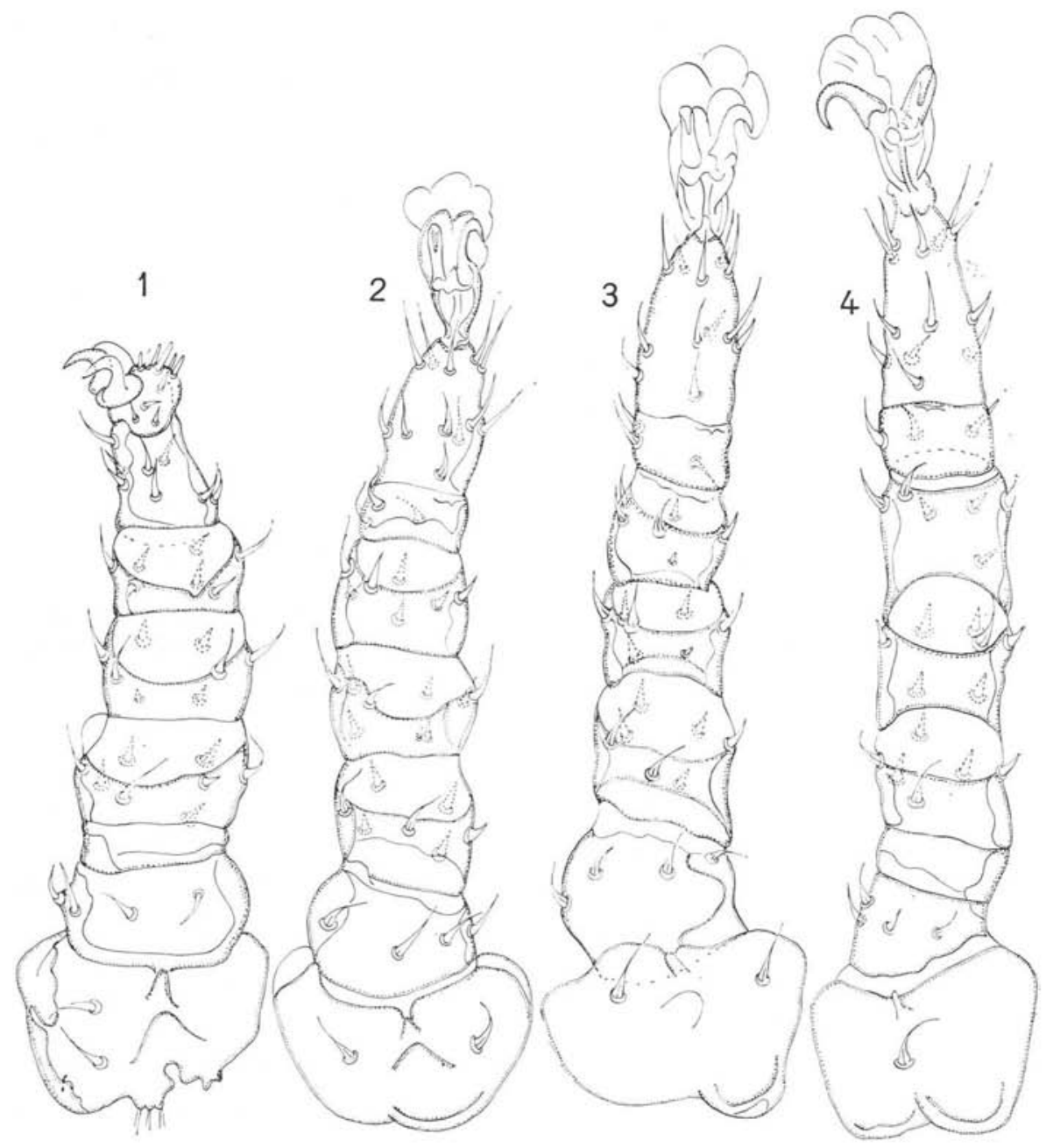

Fig. 15. - Mesonyssus minuti, femelle. Pattes 1-4.

Le bouclier anal est très large. L'orifice anal occupe le tiers de la longueur du bouclier, tandis que le cribrum occupe presque trois quarts de la longueur des bords du bouclier. Les poils sont fixés au niveau de la moitié de la longueur de l'orifice anal. Tant par la forme générale que par la position des poils, le bouclier anal de $M$. minuti diffère de $M$. gerschi.

Deutonymphe mâle. Par la transparence du tégument, à l'intérieur de la deutonymphe, on observe le corps du mâle. 


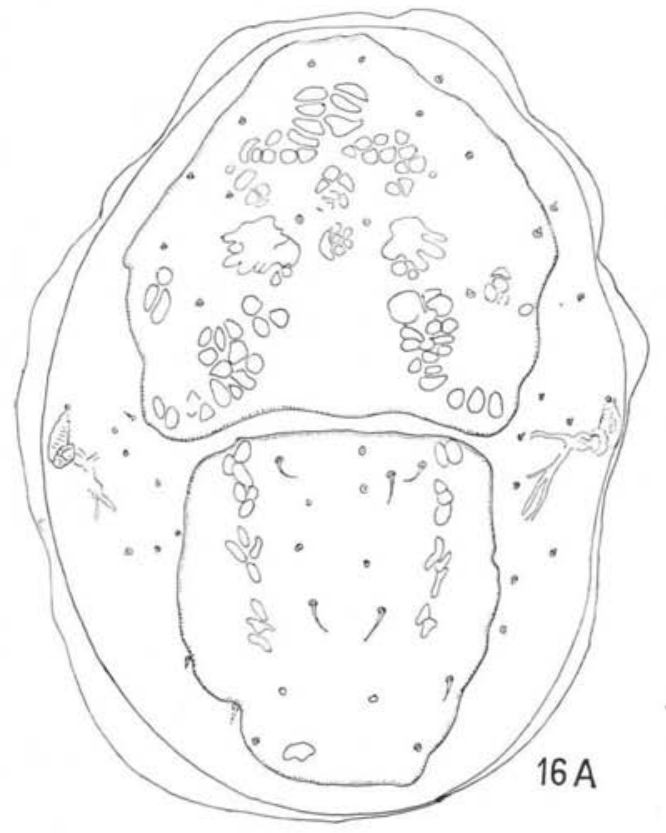

FIG. 16. - Mesonyssus minuti, mâle. A) vue dorsale, B) vue ventrale, C) bouclier anal.

FIG. 17. - Mesonyssus minuti, deutonymphe mâle. A) vue dorsale, B) gnathosoma.

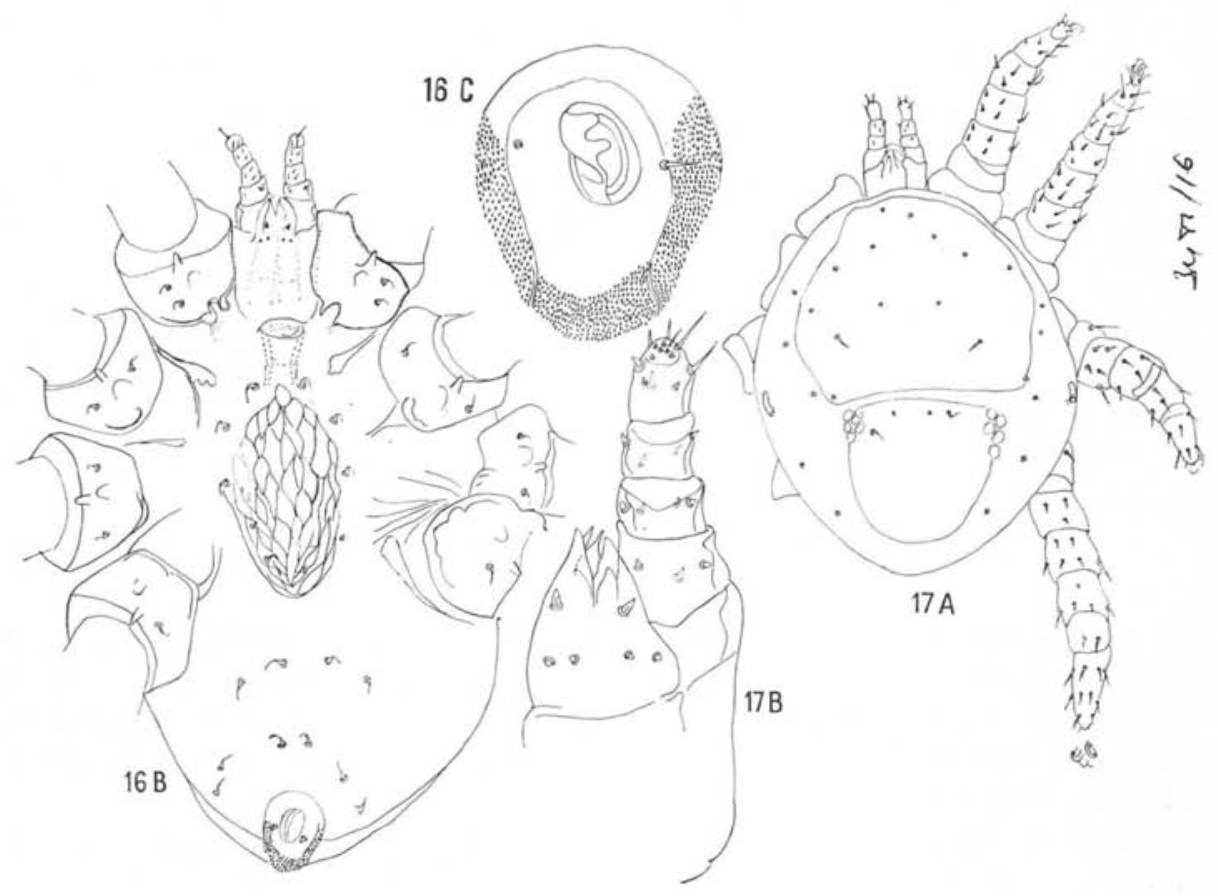


Le bouclier podosomal, dépourvu d'ornementation, est plus large que chez l'adulte et présente sept paires de poils, dont une paire de poils plus longs.

Le bouclier opisthosomal, à la différence de l'adulte, a une surface égale à la moitié du bouclier podosomal. Il porte deux paires de poils, dont une a les poils longs. L'ornementation du bouclier est limitée aux angles antéro-latéraux (fig. 17, A).

Le gnathosoma porte le même nombre de poils hypostomaux que l'adulte. Le palpe, à la différence de l'adulte, a le fémur divisé en basifémur et télofémur. La formule chétotaxique du palpe est: basifémur, 3 ; télofémur, 4 ; génual, 4 ; tibia, 7 ; et tarse 8 poils, dont un très long, un de longueur moyenne et six papilliformes.

Les pattes de la deutonymphe diffèrent des pattes de l'adulte, parce que le fémur n'est pas divisé.

Holotype et paratype femelle, allotype mâle, paratype nymphe, chez les auteurs. Terra typica : Ciric, Iași. Date : 5 mai 1968. Hôte: Ixobrychus minutus.

\section{Bibliographie}

Bregetova (A. N.), 1950. - Novîe vidî endoparaziticieskih klesci semeistva Rhinonyssidae (Gamasoidea, Acarina), D.A.N., S.S.S.R., 71 (5), 1005-1008.

-, 1964. - Some problems of evolution of the rhinonyssid mites, report presented at the first international Congress of Parasitology, Nauka, Leningrad.

—, 1967. - Rodovîe gruppirovki klescei Rinonissid, parazitov ptitz. III. Rodî Neonyssus Hirst, 1921 i Neonyssoides Hirst, 1923, Parazit. Sbor., 23, 124-137.

Butenko (M. O.), 1965. - Novîe dlia faună S.S.S.R. vidî poloctnîh klesciei ptitz. Zool. Zhurnal, 44 (1), 131-133.

-, 1969. - Zoogeograficeschii analiz fauna klescei semeistva Rhinonyssidae (Gamasoidea, Parasitiformes), Sovetscogo soiuz, Zool. Zhurnal, 48 (2), 207-211.

Cenny (V.), 1967. - Lista de los Acaros parasitos de aves reportadas de Cuba, Catalog de la fauna Cubana XX, Trabajos di divulgacion (45), 1-23.

Domrow (R.), 1965. - New laelapid nasal mites from australian birds, Acarologia, 7 (3), 430-460.

—, 1966. - Rhinonyssine nasal mite infestations in birds at Michell river Mission during the wet and dry seasons, Proc. Linn. Soc. N. Sw, 91 (3), 211-219.

FAIN (A.), 1956. - Les Acariens de la famille Rhinonyssidae Vitzthum, 1935, parasites des fosses nasales des oiseaux au Ruanda-Urundi, Rev. Zool. Bot. Afr., 53 (1-2), 131-157.

-, 1961. - Notes sur quelques Rhinonyssidés (Mesostigmata), Aracologia, 3 (3), 510521.

-, 1962. - Les rhinonyssides parasites des pigeons (Acarina, Mesostigmata), Rev. Zool. Bot. Afr., 65 (3-4), 305-323.

FEIDER (Z.), 1962. - Neonyssus hirsutus n. sp. (Rhinonyssidae, Parasitiformes), un nou parazit în cavitatea nazală la porumbelul domestic, Stud. Cercet. St. Acad. R.P.R., Iasi, 13 (1), 57-68. 
Grétillat (S.), Capron (A.) et Brygoo (R. E.), 1959. - Acariens Rhinonyssidae de Madagascar Agapornyssiae n.s. fam.; Agapornyssus n. g.; Agapornyssus faini n. sp. ; Ptilonyssus madagascariensis n. sp. et Neonyssus marcandrei n. sp. ; parasites des fosses nasales et des poumons d'oiseaux malgaches, Acarologia, 1 (4), 375-383.

RADOVSKY (F. I.), 1969. - Adaptative radiation in parasitic Mesostigmata. Acarologia, 11 (3), 450-483.

ZUMPT (F.) et Till (M.W.), 1955. - Nasal mites of birds hitherto known from the Ethiopian Region, with keys and descriptions of nine new species (Acarina: Laelaptidae). Jour. Ent. Soc. South Afr., 18 (1), 60-92. 